\title{
Genome sequence data: management, storage, and visualization
}

\author{
Jacqueline Batley ${ }^{1}$ and David Edwards ${ }^{2}$ \\ ${ }_{1}^{1}$ Australian Centre for Plant Functional Genomics, School of Land, Crop and Food Sciences and ARC Centre of \\ Excellence for Integrative Legume Research, University of Queensland, Brisbane, Australia \\ ${ }_{2}^{2}$ Australian Centre for Plant Functional Genomics and School of Land, Crop and Food Sciences, University of \\ Queensland, Brisbane, Australia
}

BioTechniques 46:333-336 (April 2009 Special Issue) doi 10.2144/000113134

Keywords: second generation sequencing; genome sequencing databases; genome visualization

Over the last few years there has been a revolution in DNA sequencing technology that has brought down the cost of DNA sequencing and made the sequencing of an increasing number of genomes both feasible and cost effective. There has also been a dramatic shift in the type of sequence data being generated, with vast numbers of short reads or pairs of short reads replacing the traditional relatively long reads produced by Sanger sequencing. These changes in data quantity and format have led to a rethinking of sequence data management, storage, and visualization, and provide a challenge for bioinformatics. The vast amount of sequence data that will be generated over the next few years will require a change in what data are stored and how users query the information.

\section{The new data}

Genome sequencing was revolutionized by the introduction of commercial pyrosequencing and the release of the Roche 454 GS20 in 2006, which could produce 20 Mbp per run. This was replaced by the GS-FLX in 2007, with a 5-fold data output improvement to $100 \mathrm{Mbp}$, which increased in 2008 to $400 \mathrm{Mbp}$ (1). Since the introduction of second generation sequencing by Roche 454 , both Illumina and Applied Biosystems (AB) have joined the market with the GAII and the SOLiD systems, respectively. Roche has continued extending average read length (currently $\sim 400 \mathrm{bp}$ with the release of the Titanium methods in 2008), while Illumina and AB have focused on producing vast numbers of reads, with read lengths currently at 75 bp (www.illumina.com) and 35 bp (2), respectively, for these systems. Manufacturers are constantly increasing output in terms of number of reads, increasing read length, as well as working to improve read quality, such that at the time of publication, it is possible to generate 20 Gbp of sequence in a single run of the Illumina GAII (www.genengnews.com/ news/bnitem.aspx? name $=48514221$ ). The rapid advances in sequence data generation suggest that we are still in the early stages of technology development in this field and that data production will continue to increase dramatically over the next few years.

It is clear that we are only at the start of next-or second-generation sequencing.
Currently, commercial companies are increasing their sequencing capability and are being joined by additional commercial sequencing technologies that will drive the market and sequencing capability much further over the coming decade. Helicos (www.helicosbio.com) has recently come onto the market with the first single molecule sequencing system, offering sequencing without the DNA amplification required for all previous systems (3). Both Pacific Biosciences (www.pacificbiosciences.com) and Nanopore technologies (www.nanoporetech.com) have commercial products in the pipeline, and DNA sequencing is expected to continue to grow at an exponential rate for several years to come. Pacific Biosciences uses single molecule real-time sequencing (SMRT) in which a DNA polymerase molecule is attached to a chip and visualized as DNA is synthesized from a template of a single-stranded DNA molecule (4). The Nanopore sequencing technology uses a thin membrane of nanopores, where the target DNA is placed on the membrane and a current is applied across the nanopore. The duration of the translocation of a polynucleotide through a nanopore channel is dependent on the DNA sequence and not the length, allowing the sequence to be determined (4).

The thousand-dollar genome is within sight $(5,6)$ and soon this cost will not only apply to resequencing, but also to de novo genome sequencing. New technologies can be applied to sequence the large and complex genomes of agronomically important plant species. It was previously considered unfeasible to sequence crops such as wheat, whose genome is six times larger than the human genome and consists predominantly of repetitive elements. The size and hexaploid nature of genomes such as wheat create significant bioinformatics challenges, and although sequence data generation is now relatively inexpensive, it may be several years before bioinformatics methods are capable of assembling large and complex genomes. Genomics technologies have moved from gene to genome sequencing and are now capable of sequencing whole environments of microorganisms. The production of this metagenomic data, however, generates another challenge for data management, because sequences cannot always be associated with specific species as has been traditional for gene and genome sequence data management.

\section{Data management and storage}

The International Nucleotide Sequence Databases consisting of GenBank (7), the DNA Databank of Japan (DDBJ) (8), and European Molecular Biological Laboratory (EMBL) (9) provide the principle repositories for DNA sequence data. In addition to hosting the text sequence data, they host basic annotation and, in many cases, the raw data from which the text sequences were derived. Although submitting the raw trace file data for Sanger sequences is 


\section{raw sequence data $\rightarrow$ text sequence data $\rightarrow$ sequence variation data}

TB-GB GB-MB MB-KB

Figure 1. The volume of data declines from processing of the raw image data to the text sequence and quality files. Further removal of redundant data by comparison with a reference genome reduces the data volume to the megabyte-kilobyte scale.

often a requirement for publication, the storage of raw data for the new technologies is problematic due to the vast size of the images. The cost of storing the gigabytes of raw data produced by each run of the Illumina GAII or AB SOLiD has been estimated to be greater than the cost of generating the data in the first place. It is now common practice to delete the raw image files once they have been processed to produce the relatively small text sequence and quality data files. While the long-term storage of the text sequence files is feasible using current tape and disc technology, maintaining the data in a readily usable form where it may readily be interrogated by users is more of a challenge. The GenBank sequence repository continues to increase in size exponentially, and searching this data using standard sequence comparison algorithms takes an extensive amount of time. Additionally, a large amount of computing power is needed to run standard tools such as BLAST. New sequence comparison tools such as Zoom (10) have been developed specifically for second-generation short read sequences; however, it may be some time before a standard comparison tool equivalent to BLAST becomes prevalent for short reads.

A greater challenge for sequence storage and management is the increasing quantity of redundant data being generated by the new sequencing technologies. Both the Illumina GAII and AB SOLiD systems were developed primarily for resequencing genomes used to identify variation between individuals. The vast majority of data produced by these resequencing experiments will be identical to the reference sequence and therefore of little primary value. There are currently projects to resequence 1000 human genomes (www.1000genomes.org), as well as multiple plant and animal varieties to identify genetic variation within species associated with phenotypic variation. The submission of complete resequence data to the international repositories would result in the storagage of highly redundant data sets, bloating the size of the database and reducing the efficiency of queries. As an increasing number of reference genome sequences become available and the cost of resequencing continues to decline, the problem of data redundancy will increase to a point where storage within the primary data repositories becomes impractical.

The theory that sequence repositories will constantly increase in size is likely to be challenged with the increasing availability of reference genome sequences. Once a reference genome sequence has been produced, users are predominantly interested in variation from this reference. While the data generated during resequencing experiments to discover this variation is vast, the valuable portionconsisting of sequence and structural variations from the reference-is relatively small in size (Figure 1). These sequence variations mostly consist of single nucleotide polymorphisms (SNPs) and insertions/deletions (InDels), and there have been moves to store and query this data outside of the main sequence repositories. This has led to databases such as dbSNP (11) and databases supporting the human and bovine HapMap projects (12). With an increase in the number of available reference sequences and continual resequencing data generation, it is expected that the value of the primary sequence repositories will decline and be replaced by increasingly sophisticated sequence variation databases that will be substantially smaller in size than the main repositories, yet offer a more valuable user experience.

\section{Data visualization}

As reference genome sequences have become available, several genome viewers have been developed to allow users to access the data. Common browsers include EnsEMBL (13), GBrowse (14), and the University of California, Santa Cruz genome browser (15). These tools offer various levels of sophistication and simplicity: some are more appropriate genome interrogation tools than others depending on the size, complexity, and completeness of the genome, as well as the need to link associated data types such as sequence variation, comparative genomes, genetic markers, gene expression, protein structure, and expression information. These viewers are increasingly including sequence variation and comparative genome analysis tools, and their implementations are becoming the primary information source for many researchers who do not wish to trawl through the underlying sequence data used to identify the sequence variation. Genome viewers and their underlying databases are becoming both the visualization and interrogation tools of choice for sequencing data. As the cost of de novo genome sequencing decreases, it will be possible to sequence any genome and not just those of model organisms. Data from the resequencing of these genomes is unlikely to be submitted to the primary sequence repositories and instead, organisms will have their own dedicated genome browsers.

The move from central sequence repositories to interactive databases of genome variation will be driven by the need to integrate the increasing quantity of related data, particularly phenotypic information associated with specific sequence variation or epigenetic status. Although major advances have been made in recent years in bridging the genome to phenome divide, it still remains one of the greatest challenges in biology today. The collection of large volumes of structured phenotypic data and its integration with the abundant genome data will add dimensions and challenges for the storage, management, and visualization of this information. New generations of genome databases, however, will allow researchers to perform comparative biology at an unprecedented level, providing insights into the foundations of life and the evolutionary processes that shape biological processes.

\section{Acknowledgments}

The authors would like to acknowledge funding support from Australian Research Council's Linkage Projects funding scheme (project no. LP0882095) and the Grains Research and Development Corporation (project no. DAN00117).

\section{References}

1. Rothberg, J.M. and J.H. Leamon. 2008. The development and impact of 454 sequencing. Nat. Biotechnol. 26:1117-1124. 
2.Morozova, O. and M.A. Marra. 2008. Applications of next-generation sequencing technologies in functional genomics. Genomics 92:255-264.

3.Harris, T.D., P.R. Buzby, H. Babcock, E. Beer, J. Bowers, I. Braslavsky, M. Causey, J. Colonell, et al. 2008. Singlemolecule DNA sequencing of a viral genome. Science 320:106-109.

4.Gupta, P.K. 2008. Single-molecule DNA sequencing technologies for future genomics research. Trends Biotechnol. 26:602-611.

5.Bennett, S.T., C. Barnes, A. Cox, L. Davies, and C. Brown. 2005. Toward the 1,000 dollars human genome. Pharmacogenomics 6:373-382.

6.Service, R.F. 2006. Gene sequencing. The race for the $\$ 1000$ genome. Science 311:1544-1546.

7. Benson, D.A., I. Karsch-Mizrachi, D.J. Lipman, J. Ostell, and D.L. Wheeler. 2006. Genbank. Nucleic Acids Res. 34:D16-D20.

8. Ohyanagi, H., T. Tanaka, H. Sakai, Y. Shigemoto, K. Yamaguchi, T. Habara, Y. Fujii, B.A. Antonio, et al. 2006. The rice annotation project database (rap-db): hub for Oryza sativa ssp. japonica genome information. Nucleic Acids Res. 34:D741-D744.

9. Cochrane, G., P. Aldebert, N. Althorpe, M. Andersson, W. Baker, A. Baldwin, K. Bates, S. Bhattacharyya, et al. 2006. EMBL nucle- otide sequence database: developments in 2005. Nucleic Acids Res. 34:D10-D15.

10.Lin, H., Z.F. Zhang, M.Q. Zhang, B. Ma, and M. Li. 2008. ZOOM! Zillions of oligos mapped. Bioinformatics 24:24312437.

11.Smigielski, E.M., K. Sirotkin, M. Ward, and S.T. Sherry. 2000. dbSNP: a database of single nucleotide polymorphisms. Nucleic Acids Res. 28:352-355.

12.Gibbs, R.A., J.W. Belmont, P. Hardenbol, T.D. Willis, F.L. Yu, H.M. Yang, L.Y. Ch'ang, W. Huang, et al. 2004. An overview of Ensembl. Genome Res. 14:925928

14. Stein, L.D., C. Mungall, S.Q. Shu, M. Caudy, M. Mangone, A. Day, E. Nickerson, J.E. Stajich, et al. 2002. The Generic Genome Browser: A building block for a model organism system database. Genome Res. 12:1599-1610.

15. Karolchik, D., R. Baertsch, M. Diekhans, T.S. Furey, A. Hinrichs, Y.T. Lu, K.M. Roskin, M. Schwartz, et al. 2003. The UCSC Genome Browser database. Nucleic Acids Res. 31:51-54.

Received 9 February 2009; accepted 10 February 2009.

Address correspondence to David Edwards, School of Land, Crop, and Food Sciences,
University of Queensland, Brisbane, QLD 4072 Australia. email: dave.edwards@uq.edu.au or Jacqueline Batley, School of Land, Crop, and Food Sciences, University of Queensland, Brisbane, QLD 4072, Australia. email: j.batley@uq.edu.au

\section{BioTechniques}

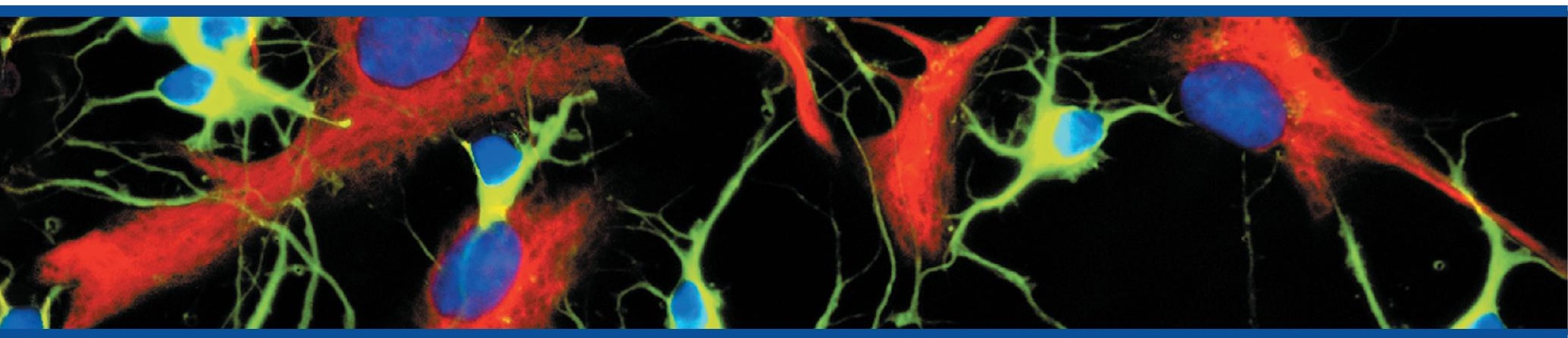

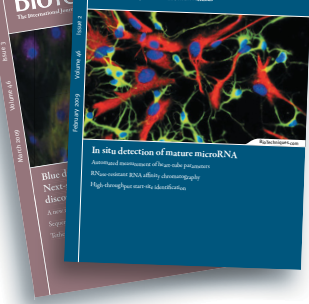

\section{Update your subscription}

Update your profile today to ensure that your copy of BioTechniques experiences no delivery disruptions.

Go to:

www.BioTechniques.com/subscribe 